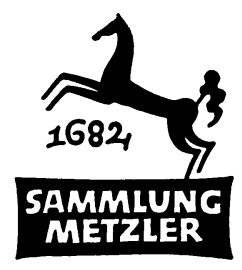

REALIEN ZUR LITERATUR ABT. D:

LITERATURGESCHICHTE 
CHARLOTTE JOLLES

\section{Theodor Fontane}

3., durchgesehene und ergänzte

Auflage

MCMLXXXIII

J. B. METZLERSCHE VERLAGSBUCHHANDLUNG

STUTTGART 
1. Auflage 1972 (1.-5. Tsd.)

2. Auflage 1976 (6.-10. Tsd.)

3. Auflage 1983 (11. -15 . Tsd.)

\section{CIP-Kurztitelaufnahme der Deutschen Bibliothek}

\section{Jolles, Charlotte:}

Theodor Fontane / Charlotte Jolles. -

3., durchges. u. erg. Aufl., (11.-15. Tsd.).-

Stuttgart: Metzler, 1983.

(Sammlung Metzler; M 114: Abt. D, Literaturgeschichte)

ISBN 978-3-476-13114-0

NE: GT

ISBN 978-3-476-13114-0

ISBN 978-3-476-04119-7 (eBook)

DOI 10.1007/978-3-476-04119-7

\section{114}

Springer-Verlag GmbH Deutschland 1983

Ursprünglich erschienen bei J. B. Metzlersche Verlagsbuchhandlung und Carl Ernst Poeschel Verlag GmbH in Stuttgart 1972/1976/1983 


\section{INHALT}

Vorwort zur dritten Auflage. . . . . . . . . . . . . . .

I. Materialien ............................ 1

1. Der Handschriftliche Nachlaß. Das Theodor-FontaneArchiv ............................ 1

2. Gesamtausgaben ...................... 5

3. Briefe und Tagebücher ................... 9

4. Bibliographische Hilfsmittel. Ausstellungskataloge...... 18

5. Monographien ........................ 19

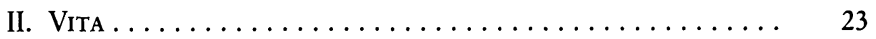

1. Herkunft .......................... 23

2. Frühe Jahre (bis 1850) .................. 25

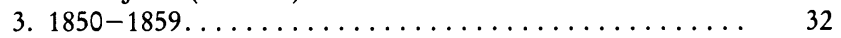

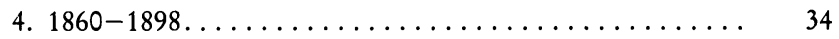

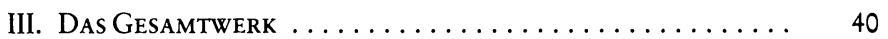

a) Gedichte.......................... 40

b) Journalismus..................... 44

1. Frühe Publizistik (1842-1850). Publizistik über England ....................... 44

2. "Wanderungen durch die Mark Brandenburg"..... 44

3. Die Kriegsbücher .................. 47

4. Kritik (Theater, Literatur, Kunst) . . . . . . . . 49

c) Romane und Novellen (in chronologischer Ordnung) 54 "Vor dem Sturm « 54 - "Grete Minde « 61 - "L'Adultera « 63 - "Ellernklipp« 65 - »Schach von Wuthenow « 67 "Graf Petöfy « 70 - »Unterm Birnbaum« 72 - "Cécilie« 74 - »Irrungen, Wirrungen « 77 - »Stine« 81 - »Quitt $\ll 82$ - „Unwiederbringlich« 85 - „Frau Jenny Treibel« 89 "Effi Briest « 92 - Die Poggenpuhls« 100 - „Der Stechlin « 102 - »Mathilde Möhring « 112

d) Kleine Prosaarbeiten ..................... 115

e) Fragmente und Entwürfe................ 115

f) Biographisches und Autobiographisches . . . . . . 118

IV. Politik und Gesellschaft - Persönlichkeit und Weltbild ........................... 126

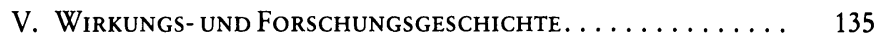

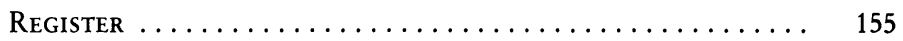


$\begin{array}{ll}\text { ADB } & \text { Allgemeine Deutsche Biographie } \\ \text { AGer } & \text { Acta Germanica } \\ \text { AION(T) } & \text { Annali. Sezione Germanica. Studi Tedeschi. } \\ \text { ASNS } & \text { Archiv für das Studium der neueren Sprachen und } \\ & \text { Literaturen } \\ \text { AULLA } & \text { Australasian Universities Language and Literature } \\ & \text { Association } \\ \text { CollGerm } & \text { Colloquia Germanica } \\ \text { DA } & \text { Dissertation Abstracts* } \\ \text { DR } & \text { Deutsche Rundschau } \\ \text { Dt. Beitr. } & \text { Deutsche Beiträge } \\ \text { DU } & \text { Der Deutschunterricht } \\ \text { DVjs. } & \text { Deutsche Vierteljahrsschrift für Literaturwissenschaft } \\ & \text { und Geistesgeschichte } \\ \text { EG } & \text { Etudes Germaniques } \\ \text { Euph. } & \text { Euphorion } \\ \text { FAP } & \text { Fontane-Archiv, Potsdam } \\ \text { FBl. } & \text { Fontane-Blätter } \\ \text { FMLS } & \text { Forum for Modern Language Studies } \\ \text { GLL } & \text { German Life \& Letters } \\ \text { GQ } & \text { German Quarterly } \\ \text { GR } & \text { Germanic Review } \\ \text { GRM } & \text { Germanisch-Romanische Monatsschrift } \\ \text { GSR } & \text { German Studies Review } \\ \text { HA } & \text { Hanser-Ausgabe der Werke } \\ \text { Hochl. } & \text { Hochland } \\ \text { Hs., Hss. } & \text { Handschrift(en) } \\ \text { IASL } & \text { Internationales Archiv für Sozialgeschichte der deutschen } \\ \text { Jb. f. Br. Lg. } & \text { Jahrbuch für Brandenburgische Landesgeschichte } \\ \text { Jb. DSG } & \text { Jahrbuch der Deutschen Schiller-Gesellschaft } \\ \text { Jb. FDH } & \text { Jahrbuch des Freien Deutschen Hochstifts } \\ \text { Jb. PK } & \text { Jahrbuch Preußischer Kulturbesitz } \\ \text { JEGP } & \text { Journal of English and Germanic Philology } \\ \text { JIG } & \text { Jahrbuch für Internationale Germanistik } \\ \text { Mag. Lit. } & \text { Magazin für die Literatur des In- und Auslandes } \\ \text { Marg. } & \text { Marginalien } \\ \text { Mh. } & \text { Monatshefte (Wisconsin) } \\ \text { ML } & \text { Modern Languages } \\ \text { MLN } & \text { Modern Language Notes } \\ \text { MLR } & \text { Modern Language Review } \\ \text { Ms. } & \text { Manuskript } \\ & \end{array}$

* Für die späteren Jahre sind den DA-Angaben die Bestellnummern beigefügt. 


$\begin{array}{ll}\text { MSp. } & \text { Muttersprache } \\ \text { NDH } & \text { Neue Deutsche Hefte } \\ \text { NDL } & \text { Neue Deutsche Literatur } \\ \text { NGS } & \text { New German Studies } \\ \text { NR } & \text { Neue Rundschau } \\ \text { NSR } & \text { Neue Schweizer Rundschau } \\ \text { NyA } & \text { Nymphenburger Ausgabe der Werke } \\ \text { NZZ } & \text { Neue Zürcher Zeitung } \\ \text { OGS } & \text { Oxford German Studies } \\ \text { OL } & \text { Orbis Litterarum } \\ \text { PMLA } & \text { Publications of the Modern Language Association } \\ \text { RG } & \text { Recherches germaniques } \\ \text { RLV } & \text { Revue des langues vivantes } \\ \text { SchwM } & \text { Schweizer Monatshefte. } \\ \text { Seminar } & \text { Seminar. A journal of Germanic Studies } \\ \text { SH } & \text { Sonderheft } \\ \text { SuF } & \text { Sinn und Form } \\ \text { Teko } & \text { Text und Kontext } \\ \text { VASILO } & \text { Adalbert-Stifter-Institut des Landes Oberösterreich. Viertel- } \\ & \text { jahrsschrift } \\ \text { WB } & \text { Weimarer Beiträge } \\ \text { Weiße Bll. } & \text { Weiße Blätter } \\ \text { WW } & \text { Wirkendes Wort } \\ \text { ZfBFr. } & \text { Zeitschrift für Bücherfreunde } \\ \text { ZfBibl. } & \text { Zentralblatt für Bibliothekswesen } \\ \text { ZfdB } & \text { Zeitschrift für deutsche Bildung } \\ \text { ZfdPh. } & \text { Zeitschrift für deutsche Philologie } \\ \text { ZfdU } & \text { Zeitschrift für deutschen Unterricht } \\ \text { Zs. } & \text { Zeitschrift } \\ \end{array}$




\section{LITERATUR}

Die folgenden Veröffentlichungen werden im Text oder in den Literaturangaben zu den Romanen oft nur mit Namen und Jahreszahl zitiert.

Aust, Hugo: Th. F.: »Verklärung«. Eine Untersuchung zum Ideengehalt seiner Werke. 1974.

Bance, Alan: Th. F.: The major novels. Cambridge 1982.

Bosshart, Adelheid: Th. Fs historische Romane. 1957.

Demetz, Peter: Formen des Realismus. Th. F. Kritische Untersuchungen. 1964. $1966^{2}$.

Fricke, Hermann: Th. F. Chronik seines Lebens. 1960.

Garland, Henry: The Berlin novels of Th. F. Oxford 1980.

Günther, Vincent J.: Das Symbol im erzählerischen Werk Fs. 1967.

Hayens, Kenneth: Th. F. A critical study. London 1920.

Kabrmann, Cordula: Idyll im Roman: Th. F. 1973.

Lukács, Georg: Der alte F. In: G. L.: Deutsche Realisten des 19. Jhs. 1951, S. 262-307.

Martini, Fritz: Th. F. In: F. M.: Deutsche Literatur im bürgerlichen Realismus. $1962 ;{ }^{2} 1964$, S. $737-800$.

Mittenzwei, Ingrid: Die Sprache als Thema. Untersuchungen zu Fs Gesellschaftsromanen. 1970.

Müller-Seidel, Walter: Th. F. Soziale Romankunst in Deutschland. 1975; $1980^{2}$.

Obl, Hubert: Bild und Wirklichkeit. Studien z. Romankunst Raabes u. Fs. 1968.

Pascal, Roy: Th. F. In: R. P.: The German novel. Manchester 1956, S. 178-214.

Reuter, Hans-Heinrich: F. 2 Bde. 1968.

Rosenfeld, Hans-Friedrich: Zur Entstehung Fontanescher Romane. Groningen/Den Haag 1926.

Rost, Wolfgang E.: Ortlichkeit u. Schauplatz in Fs Werken. 1931.

Schillemeit, Jost: Th. F. Geist u. Kunst seines Alterswerks. 1961.

Sieper, Clara: Der historische Roman u. die historische Novelle bei Raabe u. F. 1930.

Wandrey, Conrad: Th. F. 1919.

Sammelwerke, in den Literaturangaben gekürzt zitiert:

Theodor Fontanes Werk in unserer Zeit. Symposion z. 30-Jahr-Feier des Fontane-Archivs der Brandenburgischen Landes- u. Hochschulbibliothek Potsdam. 1966.

Fontanes Realismus. Wissenschaftliche Konferenz z. 150. Geb. Th. Fs in Potsdam. Vorträge u. Berichte. Im Auftr. d. Dt. Staatsbibliothek hrsg. v. Hans-Erich Teitge u. Joachim Schobeß. 1972.

Th. F. Hrsg. v. Wolfgang Preisendanz. 1973. (Wege d. Forschung Bd 381). Formen realistischer Erzählkunst. Festschrift for Charlotte Jolles. Ed. by Jörg Thunecke in conjunction with Eda Sagarra. Nottingham 1979. 
Fontane aus heutiger Sicht. Analysen u. Interpretationen seines Werkes. Hrsg. v. Hugo Aust. 1980.

Zeitschriften und Zeitungen mit Erstveröffentlichungen Fs, im Text gekürzt zitiert:

Cosmopolis. Intern. Revue. (London, Wien, Paris.)

Dabeim. Ein dt. Familienblatt, mit Illustrationen. (Leipzig.)

Das Magazin für die Literatur des In- und Auslandes. Wochenschrift der Weltliteratur. (Berlin.)

Deutsche Rundschau. (Berlin.)

Deutschland. Wochenschrift $\mathrm{f}$. Kunst, Literatur, Wissenschaft $\mathrm{u}$. soziales Leben. (Glogau.)

Die Gartenlaube. Illustr. Familienblatt. (Leipzig.)

Nord und Süd. Eine dt. Monatsschrift. (Berlin.)

Pan. (Berlin.)

Úber Land und Meer. Illustr. Wochenblatt. (Stuttgart.)

Universum. Illustrierter Hausschatz f. Poesie, Natur u. Welt, Literatur, Kunst u. Wissenschaft. (Dresden.)

Vom Fels zum Meer. Illustr. Zeitschr. f. d. dt. Haus. (Stuttgart.)

Vossische Zeitung. Königlich privileg. Berlinische Ztng von Staats-u. gelehrten Sachen. Vossische Zeitung. (Berlin.)

Westermanns Illustrierte Deutsche Monatshefte. (Braunschweig.) 


\section{VORWORT ZUR DRITTEN AUFLAGE}

"Kein Autor des 19. Jahrhunderts hat im Berichtszeitraum so sehr im Mittelpunkt des Interesses gestanden wie Theodor Fontane «, heißt es im Forschungsbericht zur Deutschen Literatur des 19. Jabrbunderts, der 1980 im Jahrbuch für Internationale Germanistik herausgekommen ist. Der Bericht umfaßt die Jahre 1960 bis 1975. Jetzt allerdings hört man manchmal, daß die Fontane-Renaissance, die in den sechziger Jahren mit dem Beginn neuer Editionen des Werkes und der Briefe sowie mit einer monumentalen Biographie aus neuer Sicht ihren Anfang nahm, im Abflauen begriffen oder gar vorüber sei. Dies ist keineswegs der Fall. Im Gegenteil, eine gewisse Konsolidierung hat stattgefunden. Allein in der Zeit zwischen Anfang 1976 und Anfang 1983 sind in dieser dritten Auflage an die vierhundert neue Titel zu verzeichnen.

Die Werkausgaben sind zum Teil abgeschlossen oder nähern sich dem Abschluß, nur die Arbeit an der zuletzt begonnenen AufbauAusgabe wird sich noch über Jahre erstrecken. Eine umfangreiche Auswahl aus Fontanes Briefwerk ist soeben erschienen, ebenso die Korrespondenz mit Storm; weitere Briefwechsel sind geplant oder schon in Bearbeitung. Immer wieder werden bisher noch unbekannte kleine Entwürfe und Aufzeichnungen aus dem Nachlaß herausgegeben. Viel solide kritische und philologische Arbeit steckt in den Anmerkungen und im Anhang der Werkausgaben sowie auch in den „Erläuterungen und Dokumenten“ zu Fontanes Romanen, die Reclam in Stuttgart herausbringt.

Eine neue kritische Literatur befaßt sich mit der Aufnahme Fontanes in den audiovisuellen Medien, die sich in immer größerem Maße und auch mit kritischer Einsicht seiner Romane annehmen. Die Fontane-Renaissance, die im wissenschaftlichen Bereich eingesetzt hatte, ist nicht ohne Einfluß auf Rundfunk, Fernsehen und Film geblieben, die dem Dichter eine weitere Anhängerschaft und einen größeren Leserkreis und sogar eine gewisse Massenwirkung verschaffen.

Auch Übersetzungen Fontanescher Romane in fremde Sprachen haben in den letzten Jahren zugenommen, und in der hier vorgelegten Literatur finden wir Arbeiten, die sich speziell mit dem Problem der Übersetzung Fontanes beschäftigen. Es ist jedoch noch lange nicht Fontanes ganzes Romanwerk im Ausland bekannt, und die Verbreitung läßt sich keineswegs mit der anderer europäischer Romanciers des 19. Jahrhunderts vergleichen oder etwa der Thomas Manns.

Doch wächst Fontanes Stellung in der internationalen Germani- 
stik und Komparatistik zunehmend. Zahlreich sind die Dissertationen, die sich mit den verschiedensten Aspekten von Fontanes Kunst befassen. Sein Werk übt zweifellos eine starke Anziehungskraft auf die jüngere internationale Forschergeneration aus, vor allem in den deutschsprachigen Ländern und in der angelsächsischen Welt. Die meisten Untersuchungen, ob in Buchform oder in Artikeln, von jüngeren Wissenschaftlern oder erfahrenen Kritikern geschrieben, befassen sich mit dem Werk, weniger mit der Persönlichkeit und Entwicklung des Dichters, obwohl auch hier einiges erarbeitet worden ist. Man vermerkt die Ambivalenz in Fontanes Kunst und in seinen Anschauungen, aber die Persönlichkeit Fontanes wird immer noch zu undifferenziert gesehen. Untersuchungen über das Verhältnis zu seinen Freunden, wie zum Beispiel zu Paul Heyse (G. Friedrich, 1980), erlauben uns tiefere Einblicke in seinen durchaus komplexen Charakter; eine Arbeit über Lepel-Fontane ist im Entstehen und mag weitere Einsichten bringen.

Die Analyse der literarischen Vermittlungsprozesse rückt immer mehr ins Zentrum literarhistorischer Arbeit. Wir besitzen jetzt die Korrespondenzen mit den wichtigsten seiner Verleger und Redakteure: Cotta, Hertz, Wilhelm Friedrich, Brockhaus, Rodenberg und Kletke, sowie eingehende soziologische Untersuchungen über Fontanes Erzählwerk in den Presseorganen unter den Marktbedingungen seiner Zeit. Die zuletzt erschienene Arbeit dieser Art ist die über den Verlag von Wilhelm Hertz (1981). Gegenüber den Einzelaspekten bietet eine literatur-soziologische Studie eine Gesamtschau über Fontane und das literarische Leben seiner Zeit (Liesenhoff, 1976). Auf diesem Gebiet ist also in den letzten Jahren Bedeutendes geleistet worden.

Auch wird die Rezeption Fontanes rege erforscht, die zeitgenössische sowie die spätere bis 1914. Die Rolle, die Thomas Mann in dieser Hinsicht gespielt hat, wird dabei immer wieder hervorgehoben. Neben der kritischen Rezeption werden neue Aspekte der dichterischen Rezeption aufgedeckt; so wurde - neben Thomas Manns Romanwerk - sehr unerwartet eine Rezeption Fontanes bei Hofmannsthal festgestellt und eingehend untersucht (K. Mommsen). Diese Problemstellung wird weitergeführt bis zur Gegenwart, bis zur Rezeption im Werk einiger DDR-Schriftsteller.

Was die Hermeneutik anbetrifft, so finden wir neben der traditionellen philologischen Hermeneutik eine Unmenge von interpretatorischen Methoden, die in der internationalen Fontane-Forschung ebenso mannigfaltig sind wie in der neueren Literaturwissenschaft überhaupt. Es überwiegen gesellschaftskritische Fragestellungen und solche nach dem Wesen des Fontaneschen Realismus. Interes- 
sant ist, daß, wie verschieden auch die methodischen Ansätze sein mögen, immer Fontanes gesellschaftskritische Intentionen betont werden. Die junge Generation hat dafür einen viel schärferen Blick entwickelt als die ältere Forschung.

Ein dem Realismus anscheinend widersprechendes irrationales Element in Fontanes Romanwerk wird von mehreren Forschern wahrgenommen, und die kritische Erarbeitung des Melusinenmotivs führte dazu, in vielen der Fontaneschen Frauengestalten Züge elementarischer elbischer Wesen zu erblicken. Das durchzieht fast das ganze Erzählwerk von Marie Kniehase über Grete Minde, Hilde in Ellernklipp, Cécile, Ebba von Rosenberg in Unwiederbringlich, bis zu Effi Briest und Melusine von Barby; auch Victoire aus Schach von Wuthenow wird darin eingeschlossen. Dies führt zur Aufspürung von mythischen Elementen bei Fontane, die bis ins einzelne analysiert werden. Es besteht hier eine gewisse Gefahr, daß bei Überbetonung des Elementaren die Rolle, die der Frau bei Fontane im sozialen Bereich zukommt, unterschätzt wird, und es ist gut, daß andere Arbeiten dies zurechtrücken.

Das Subtile in Fontanes Kunst verlockt zu immer neuen Interpretationen, die sich, auch mit Zuhilfenahme von Psychoanalyse und Psychosymbolik, der verschiedensten literarwissenschaftlichen Methoden bedienen. So wird einerseits aufgezeigt, wie minutiöse Textanalysen nach der Art der explication de texte die Kunst Fontanes transparent machen können, ohne Suche nach Symbolen, denn Fontane sei kein verschlüsselter Schriftsteller, heißt es (H. Remak). Neben dieser Meinung stehen dagegen die Versuche vor allem jüngerer Kritiker, den Geheimnissen des Künstlers durch eigene subtile Erfindungskraft auf den Grund zu gehen. Die jugendliche Passion Fontanes für das Versteckspiel, auf das in mehreren Arbeiten eingegangen wird, verleitet zu einer "Schatzsuche " auch in dem anscheinend so einfach-klaren und doch so kunstvollen Gewebe der Fontaneschen Romane. Man begnügt sich nicht mehr mit dem Verstehenwollen des Werkes und dem Begreifenwollen des "Wie“ der Aussage und der "Finessen“, an denen der Dichter selber seine Freude hatte, sondern rückt weiter "hinter" das Werk, bemüht, den schöpferischen Akt zu durchleuchten. Dabei treten dann oft, bei ähnlicher Absicht, völlig verschiedene Resultate zutage. Von einigen Kritikern als Spekulationen abgelehnt, regen sie andere wieder zu neuen Höhenflügen an. In einer erst kürzlich erschienenen Arbeit über Fontanes "Finessen " wird von einer "bedenklichen Tendenz" in der neueren Fontane-Forschung gesprochen, die als „Safari-Methode der Literaturkritik“ bezeichnet wird (Guthke, 1982). Die Spanne $\mathrm{zwischen}$ den verschiedenen interpretatorischen und analytischen 
Ansätzen ist unendlich weit geworden. Die Unsicherheit der ästhetischen Wertungen zeigt sich vor allem auch in der dauernden Aufund Abwertung der einzelnen Romane.

Fontane erzählt in seinen Kinderjabren von seiner Versteckspielpassion und seinem stundenlangen Verschwinden auf dem Heuboden, in der „glückseligen Vorstellung “: „Und wenn sie dich suchen bis an den Jüngsten Tag, sie finden dich nicht." Sollten diese Worte des Dichters nicht vielleicht Bezug haben auf die vielen und verschiedenartigen Bemühungen, ihn zu "finden"?

Die Anlage der dritten Auflage ist nicht verändert worden, da sie sich als nützlich erwiesen hat; so wird für diejenigen, die mit der Realiensammlung schon vertraut sind, in der Benutzung keine Umstellung nötig sein. Was an "Materialien" neu hinzugekommen ist, wie einige wieder aufgetauchte Manuskripte, ist an den betreffenden Stellen verzeichnet. Die bibliographischen Rubriken sind durch Veröffentlichungen bis Anfang 1983 erweitert worden, aber nur in repräsentativen Fällen wurde im Text auf die Neuerscheinungen aufmerksam gemacht. Der Text wurde nur dann verändert, wenn die Entwicklung es nötig machte, wie vor allem im Falle der EffiBriest-Forschung.

Bei der Zusammenstellung der neuen Titel waren mir das Institute of Germanic Studies in London und seine Mitarbeiter wieder eine große Hilfe. Ihnen sowie Barbara Mockert, die mir auch bei dieser Auflage zur Seite gestanden hat, schulde ich Dank.

Charlotte Jolles

London, im Frühjahr 1983 